\title{
Editorial: Remote Sensing for Environmental Studies
}

\author{
Guest Editor: Ni-Bin Chang* \\ Department of Civil and Environmental Engineering, University of Central Florida, Orlando, Florida 32816-2450, USA
}

\begin{abstract}
The need for next-generation sensors for exploring the dynamics and interactions between natural systems and human society is greater now than ever in the field of environmental informatics. These new and existing optical and microwave sensors should have enhanced the detection capabilities, preferably stand-off, and be sufficiently rugged to provide detection to warn to environmental change in diverse and extreme environments. Data streams from such optical and microwave remote sensing sensors, either air-borne and/or spaceborne must be integrated and processed so that the data is realized at the level of the user in a simple and clear format to allow for rapid decision making by environmental managers. Though many technology barriers currently exist to achieve these desired sensor features, it is believed that the combined insights, know-how and capabilities of the cross-disciplinary teams of scientists and engineers in the world can overcome several of these barriers to achieve the development of required detection capabilities for environmental uses based on the current satellites available. It is the aim of this special issue to present the multidisciplinary studies, which demonstrate some of the success in the field of remote sensing for the environment. This special issue therefore collects six papers to explore several challenges both in the atmospheric and terrestrial environments, entitled as below:
\end{abstract}

- Wavelet-based classification of remotely sensed images: A comparison of different feature sets in an urban environment
- Fluvial Geomorphologic and Hydrodynamic Assessment in the Tidal Portion of the Lower Rio Grande River, US-Mexico Borderland

- Spectral Enhancement and Automated Extraction of Potential Sinkhole Features from NAIP Imagery -- Initial Investigations

- Evaluating Satellite-based Measurements for Mapping Air Quality in Ontario, Canada

- Detection of Coastal Region Sea Ice Decay from Orthorectified RADARSAT-1 ScanSAR Imagery: A Case Study of Bering Strait and Norton Sound, Alaska

- Remote sensing derived crop coefficient for estimating crop water requirements for irrigated sorghum in the Gezira scheme, Sudan

Articles in this special issue combine the confluence of ideas and expertise in the area of Environmental Informatics from diverse points of view in dealing with unique environmental management issues. Each of these articles represents a comprehensive study of recent developments in their field. All papers were peer reviewed in accordance with required journal standards. It is indeed a great pleasure for me to see so many eminent scholars from different countries present and share their precious experience for this special issue. Finally, I wish to thank every author and reviewer for his or her enthusiastic contributions to this special issue.

\footnotetext{
*Corresponding author: nchang@mail.ucf.edu
} 